tion the popliteal lymph node began to enlarge due to lymphadenitis. The normal lymph node has a volume of about $3 \mathrm{~mm}^{2}$ but after a few repeat infections can become as much as $12,000 \mathrm{~mm}^{2}$, an increase of some 4,000 times. Post mortem and xeroradiographic studies have shown that lymphangitis develops after about four weeks and increase in severity with increasing numbers of infections.

In people the first signs of clinical disease are fevers, lymphadenitis and lymphangitis, usually occurring together and usually detected some years after birth. Filarial fever may be accompanied by rigor, chills and sweating, it persists for 2-7 days and typically occurs 3-6 times per annum. We did not measure the temperature of our cats on a daily basis so cannot be sure that filarial fevers occur in them.

In people lymphadenitis is usually acute with enlarged painful lymph nodes but we would predict that non-painful swellings occur much earlier and are ignored. Acute lymphangitis also occurs with retrograde, cord-like, painful swellings of the lymph nodes. Acute lymphangitis occasionally results in abscess formation along the line of the lymphatic and sometimes these abcesses breakthrough to the surface to produce a painful ulcer. These occur especially on the inside of the thighs. Scars from these ulcers are often seen in patients from endemic areas. In our cats some showed lymphangitis of the lymphatics on the inside of the thigh and sometimes these developed into scaby lesions very like those seen in people.

In people filariasis is a progressive disease as long as infection continues. Repeated acute attacks commonly result in chronic lymphadenitis which produces enlarged lymph nodes but reduced or no tenderness. In cats the lymph nodes shrink almost back to the size seen in normal cats.

In some people Grade I lymphoedema develops after repeated attacks of acute disease. This is characterised by heathly skin overlying a soft pitting oedema. Grade I lymphoedema is intermittent and, after a few weeks, regresses completely without residual swelling between episodes.

Grade II lymphoedema follows repeated attacks of acute lymphangitis and Grade I lymphoedema. Grade II lymphoedema is chronic with slight or no pitting and much deep fibrosis. The skin is healthy but does not pinch up. This may develop into Grade III lymphoedema which exhibits as solid, non-pitting oedema. Some of our cats developed soft oedema which is comparable to Grade I in people. This is usually self resolving and of short duration but one cat developed apparently permanent lymphoedema.

In men the genitalia are more commonly affected than any other area. Funiculitis and epididymo-orchitis with local pain and scrotal oedema are common. The most common manifestation of clinical Wuchereria bancrofti disease is hydrocoel. This is a fluid accumulation in the tunica vaginalis causing the testes to appear grossly enlarged. Many of our experimental cats were female and the males were castrated so we could not see this phenomenon, and, anyway, Brugia malayi does not cause hydrocoel in men.

We believe that the early development of pathology in cats is extremely similar to that of people and repeated infected cats represent a way of studying the sequential development of pathology and can provide answers to such questions as the effects of macrofilaricidal drugs and vaccination on the development of filarial disease.

\section{HUMORAL IMMUNE RESPONSES IN CATS REPEATEDLY INFECTED WITH Brugia pahangi}

BALDWIN C.I.*, MEDEIROS F.*, MALECELA M.* AND

DENHAM D.A.*

KEYWORDS : immunoglobulins. isotypes. feline. cat. filariasis. Brugia pahangi. immune responses.

ats repeatedly infected with Brugia pahangi exhibit many of the parasitological and pathological responses seen in humans infected with Brugia malayi or Wuchereria bancrofti the causative agents of human lymphatic filariasis (Denham and Fletcher, 1987 ; Denham et al., 1992).

In this study we have followed the humoral immune responses of cats, from each of the 4 parasitological groups described by Denham et al. (1992) (Figure 1), over 2-3 years. The results from this study may be summarised as follows : Group I : Cats in this Group are not immune to adult worms or microfilariae $(\mathrm{mf})$ although it has been reported that after 10-20 re-infections an immunity to L3 is generated (Denham et al., 1972). Cats in this group produce low levels of total IgG1 and IgG3 and high levels of IgG2 throughout the infection. There are high levels of filarial specific IgG1 and IgG2 throughout the infection with low levels of IgG3 (Figure 2). Filarial specific IgE is very rarely detected in this group.

Group II : These cats become immune to the adult worm after the first year of repeat infection. There is no immunity to $\mathrm{mf}$. These cats have an IgG profile similar to that of Group I cats (Figure 2). The exception is that these cats produce high levels of filarial specific IgE from about week 30 (Figure 3a).

Group III : These cats become immune to $\mathrm{mf}$ within the first year of infection. They are not immune to adult worms. We assume that these cats possess an immunity similar to that seen in Group I cats against L3. The main difference in IgG responses in this group as compared to Group I is that high levels of filarial specific IgG3 are produced when the cats become amicrofilaraemic (Figure 2). These high titres fall shortly after the $\mathrm{mf}$ are cleared. At late time points there is also a fall in IgG2 titres whilst IgG1 titres remain high. Filarial specific IgE cannot be detected in these cats.

Group IV : These cats become immune to both the $\mathrm{mf}$ and adult stages within the first year of infection. They produce, both, high levels of filarial specific IgG3 and IgE at the time the filarial worms are killed. In all Group IV cats the filarial specific IgG3 declines shortly after the death of the worms and IgG1 becomes the dominant antibody (Figure 2). We also see two types of IgE response where some cats produce high levels of IgE for a long time after the adult worms are killed (high responder) (Figure 3b) and some produce high levels of IgE only when the adult worms are killed (low responder) (Figure 3c).

The mechanisms of immunity to L3 are unknown in any of the four Groups described here. In Group I cats we suggest that IgG2 acts as a blocking antibody. This may be by one of two mechanisms. Firstly it has been shown that $\mathrm{mf}$ from

* London School of Hygiene and Tropical Medicine, Keppel Street, London, WC1E 7HT, England. 